\title{
The thermodynamic and the continuum limit of meson screening masses
}

\author{
O. Kaczmarek, E. Laermann, M. Müller* \\ Fakultät für Physik, Universität Bielefeld, D-33615 Bielefeld, Germany \\ E-mail: okacz, edwin, mmueller@physik.uni-bielefeld.de
}

\begin{abstract}
We present results on the thermodynamic and continuum limit of meson screening masses in the deconfined phase, using standard staggered and non-perturbatively clover-improved Wilson fermions in the quenched approximation with light quark masses.

For two temperatures, $1.5 \mathrm{Tc}$ and $3.0 \mathrm{Tc}$, it is found that on finite lattices screening masses differ between the actions. We study if both actions reproduce the same masses in the continuum by employing different methods of extrapolation to the thermodynamic and continuum limit.
\end{abstract}

31st International Symposium on Lattice Field Theory - LATTICE 2013

July 29 - August 3, 2013

Mainz, Germany

\footnotetext{
* Speaker.
} 


\section{Introduction}

Meson screening masses, since long, have been calculated in finite temperature QCD, being a measure of hadronic quark-gluon plasma excitations. The methods employed reached from hard thermal loop calculations [1] to approaches by dimensional reduction [2] to lattice simulations, see e.g. $[3,4]$ for the latest studies with dynamical staggered quarks.

In finite temperature lattice calculations meson screening masses can be determined with high precision. However, it was observed that on finite lattices the results obtained with different discretization schemes, the Wilson action with non-perturbative clover improvement and the standard staggered action in particular, did not agree. These deviations are assumed to be caused by different discretization effects. Although both actions share that to leading order the finite lattice spacing effect is $\mathrm{O}\left(a^{2}\right)$, the pre-factors may differ. Differences can also emerge due to varying finite volume effects.

Here we present a systematic study in quenched lattice QCD to investigate this assumption by carrying out the thermodynamic as well as the continuum limit for both actions.

\section{Lattice setup}

At two temperatures in the deconfined phase, 1.5 $T_{c}$ and 3.0 $T_{c}$, meson screening masses have been calculated for the pseudoscalar (PS), scalar (S), transverse vector (V) and transverse axialvector (AV) channel.

For the low temperature, a set of five aspect ratios $N_{\sigma} / N_{\tau}=2,3,4,6,8$ allowed to take the thermodynamic limit, while four temporal lattice extents $N_{\tau}=8,10,12,16$ were selected for the continuum extrapolation. For the high temperature, a reduced set of four aspect ratios $N_{\sigma} / N_{\tau}=2,3,4,8$ and three temporal extents $N_{\tau}=8,12,16$ proved to be sufficient for the analysis. Ensembles of 100 to 350 gauge field configurations have been analyzed at each lattice size.

For both fermion actions, the non-perturbatively improved clover action [5] and the standard staggered discretization, the investigation was carried out for light (valence) quark masses, $m_{q, \overline{M S}}(\mu=2 \mathrm{GeV})<25 \mathrm{MeV}$. At and below this value no noticeable quark mass effect on the screening masses was found.

\section{Meson screening masses}

The meson screening masses are extracted from meson correlators, evaluated along one of the spatial axes (chosen as $n_{z}$ in the following) and projected to zero 'momentum' ( $p_{x}=p_{y}=p_{\tau}=0$ ). On a finite lattice with periodic boundary conditions the correlator for Wilson-type fermions reads

$$
G^{\mathrm{Wilson}}\left(n_{z}\right)=\sum_{i} A_{i}\left(e^{-m_{i} n_{z}}+e^{-m_{i}\left(N_{\sigma}-n_{z}\right)}\right)=\sum_{i} A_{i}^{\prime} \cosh \left(\left(N_{\sigma} / 2-n_{z}\right) \cdot m_{i}\right) .
$$

The steepness of the exponential fall-off is given by the (screening) masses $m_{i}$ of the different states. The ground state $m_{0}$ dominates the correlator for large separations $n_{z}$. For fermions of staggered type the situation is slightly more complicated due to the presence of socalled parity partners that 

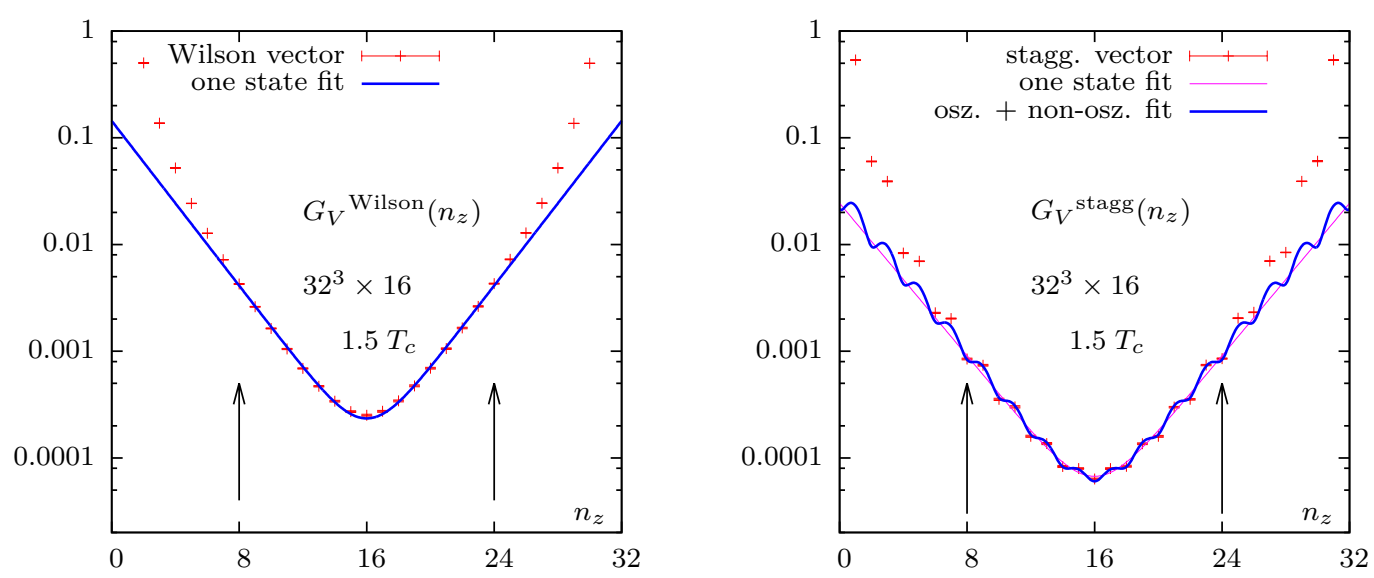

Figure 1: A fit to the vector correlation function measured on a $32^{3} \times 16$ lattice at $1.5 T_{c}$. Wilson correlator (left) fitted with eq. (3.1), and staggered correlator (right) with an additional oscillating ground state contribution through eq. (3.2). The arrows mark the fit window $\frac{1}{4} N_{\sigma} \ldots \frac{3}{4} N_{\sigma}$.

contribute with an oscillating part $\sim(-1)^{n_{z}}$ to the correlator,

$$
G^{\text {stagg. }}\left(n_{z}\right)=\sum_{i}\left(A_{i}^{\prime, \text { no }} \cosh \left(\left(N_{\sigma} / 2-n_{z}\right) \cdot m_{i}^{\mathrm{no}}\right)+(-1)^{n_{z}} A_{i}^{\prime, \text { os }} \cosh \left(\left(N_{\sigma} / 2-n_{z}\right) \cdot m_{i}^{\mathrm{os}}\right)\right)
$$

A convenient way to extract the screening masses is to fit the $A_{i}^{\prime}, m_{i}$ in eqs. (3.1) and (3.2) to a correlator measured on the lattice. These fits can be performed by taking a varying number of states into account. In the following, one-state fits refer to taking into account only the ground state contribution(s) $m_{0}$, and two-state fits refer to taking into account also one excitation. For two-state staggered fits, only the dominant, either oscillating or non-oscillating part is fitted with two states contributing.

\section{One-state fits}

Fitting eqs. (3.1) and (3.2) to data, care has to be taken in selecting an appropriate fit window $n_{z}^{\prime} \ldots N_{\sigma}-n_{z}^{\prime}$. Varying the fit window can be used to find a plateau, where the mass will level off indicating that the fit has stabilized to a ground state, see fig. 3. However, on smaller lattices no such plateau is found, since the necessary separation $n_{z}^{\prime}$ from the source can not be chosen large enough.

Thus, a different approach - inspired by the analysis of effective masses in the high temperature, free limit - is used: A fixed, consistent value of $n_{z}^{\prime}=N_{\sigma} / 4$ was chosen for all lattices, motivated by ground state plateau onset on larger lattices. On lattices with smaller aspect ratios, these fits will inevitable pick up contributions from exited states and merge them into a higher mass $m_{0}$. The contributions from these higher states are removed in the next step of the analysis, by extrapolating towards the thermodynamic limit. 

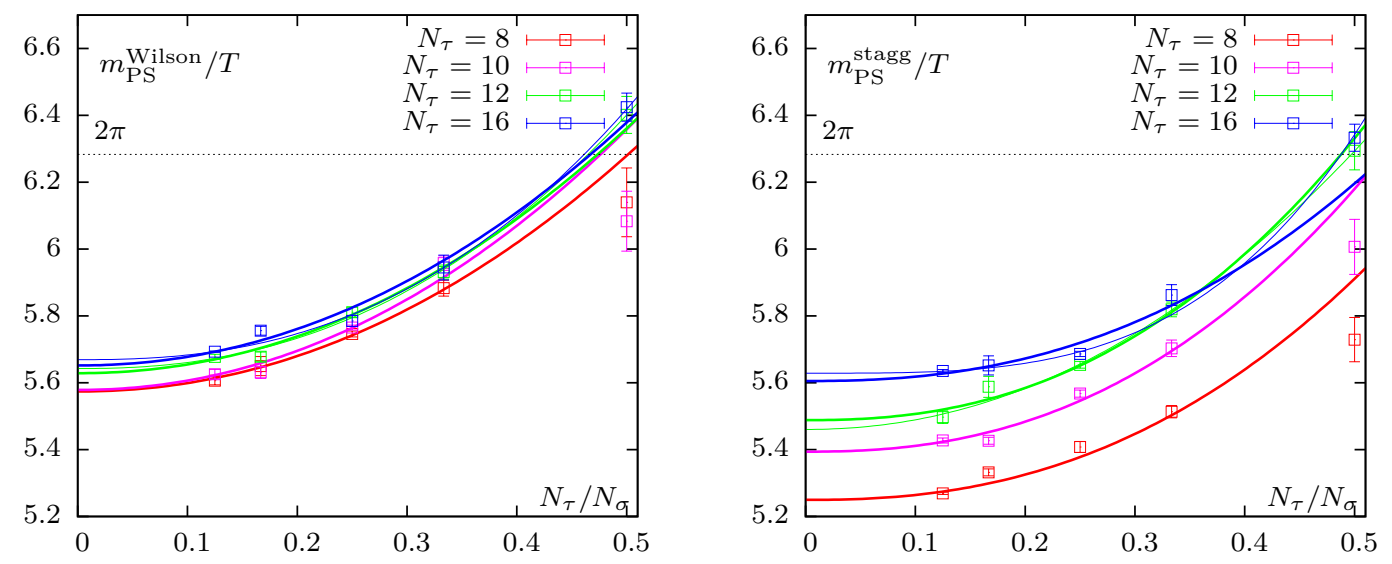

Figure 2: Thermodynamic limit obtained by fitting eq. (5.1) to Wilson (left) and staggered (right) pseudoscalar meson screening masses at $1.5 T_{c}$. The thick lines are obtained from a full fit with one shared exponent $c$ for all lattice spacings. The thin lines are obtained by fits with a dataset limited to $N_{\tau}=12$ and $N_{\tau}=16$ to crosscheck the assumption that the exponent $c$ is independent of the lattice spacing. The dotted line at $2 \pi$ marks the free theory limit.

\section{Thermodynamic limit}

An ansatz for the thermodynamic limit $N_{\sigma} \rightarrow \infty$ now has to be found and motivated: For the free theory, the leading dependence on $N_{\sigma}$ for the screening masses extracted as explained above is linear in $1 / N_{\sigma}$. From zero temperature simulations, the mass at finite volume is found to scale with the third power of the spatial lattice extent [6]. For finite temperature, it is therefore sensible to choose

$$
m_{N_{\sigma} / N_{\tau}}=m_{N_{\sigma} \rightarrow \infty / N_{\tau}}\left(1+b_{N_{\tau}} \cdot\left(N_{\tau} / N_{\sigma}\right)^{c}\right)
$$

as an ansatz for the fit, with three free parameters $m_{N_{\sigma} \rightarrow \infty / N_{\tau}}, b_{N_{\tau}}$ and $c$.

The thermodynamic limit $m_{N_{\sigma} \rightarrow \infty / N_{\tau}}$ is still subject to finite spacing effects, therefore a separate value has to be obtained for every temporal lattice extend $N_{\tau}=8,12, \ldots$ However, a combined fit can be performed to reduce the total number of free parameters and stabilize the fit: As indicated by the index, $b_{N_{\tau}}$ in eq. (5.1) is allowed to vary for different $N_{\tau}$. The exponent $c$, on the other hand, should only depend on the temperature and thus is shared among all $N_{\tau}$.

In fig. 2 the result of these fits can be found for the pseudoscalar at $1.5 T_{c}$. The figure also shows (as thin lines) two fits in which the exponent $c$ was determined with the 5 data points from $N_{\tau}=12$ and $N_{\tau}=16$ lattices only, in order to crosscheck whether the procedure of fixing the exponent to be independent of the lattice spacing is correct.

\section{Two-state fits}

A second approach to extract the ground state is to fit the correlator to eqs. (3.1) and (3.2) including one excitation. In fig. 3, different fit window sizes have been tested for both one-state and two-state fits. As expected, the mass found by one-state fits is clearly influenced by the fit window size, as it raises towards the source. For the two-state fits, the exited state $m_{1}$ is found to 

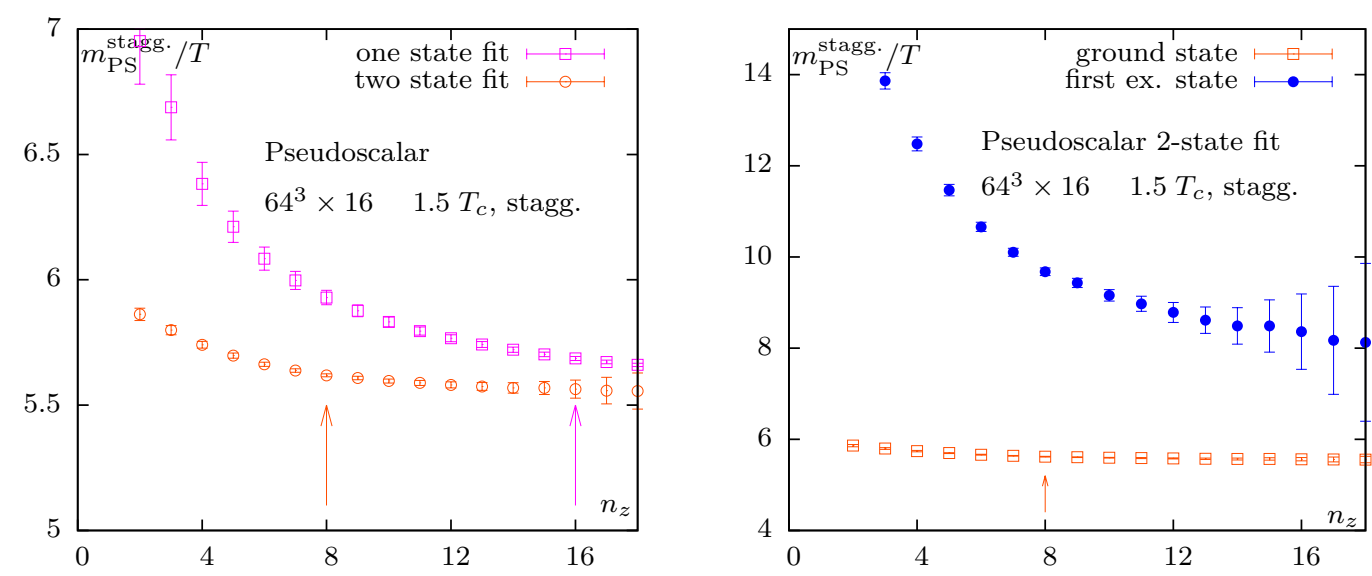

Figure 3: Staggered pseudo-scalar screening masses, obtained at $1.5 T_{c}$ on $64^{3} \times 16$ lattices, for different sizes $n_{z} \ldots N_{\sigma}-n_{z}$ of the fit window. Left: The ground state results of the one- and two-state fits are compared, where the one-state fits show a much stronger dependence on the fit window size. Right: The ground state and the exited state of the two-state fits are compared, showing that when increasing the fit window size, higher contributions mainly enter into the existed state, while the ground state is mostly uneffected. The arrows mark the fit window position $n_{z}$ chosen for the analysis.

absorb the higher contributions, while the ground state is stable over a wide region of fit window sizes. This motivates to set $\frac{1}{2} N_{\tau} \ldots N_{\sigma}-\frac{1}{2} N_{\tau}$ as a fit window for two-state fits, to ensure a constant physical distance from the source for all aspect ratios and lattice spacings.

The procedure fails for very small aspect ratios $\left(N_{\sigma} / N_{\tau}=2,3\right)$, where the system is too small to show a clear ground state. A minimal aspect ratio of $N_{\sigma} / N_{\tau}=4$ seems to suffice for larger $N_{\tau}=12,16$, while $N_{\sigma} / N_{\tau}=6,8$ give more precise results, especially on coarse lattices $N_{\tau}=8,10$.

For these larger aspect ratios, the fit results show no clear dependence on the lattice volume, thus they are averaged. For the pseudo-scalar and scalar channels as well as all staggered channels, results from the two-state fits are compatible with the thermodynamic limit of one-state fits shown in fig. 2. For the Wilson vector and axial-vector channel, results from the one-state fits are somewhat lower then from the two-state fits. Looking ahead on the final comparison of both actions, the thermodynamic limit through eq. (5.1) probably overestimated the finite volume effects.

\section{Continuum limits}

After the thermodynamic limit or the ground state mass from a two-state fit have been obtained, they have to be extrapolated to the continuum.

Both the clover-improved Wilson action as well as the standard staggered fermion action show $\mathrm{O}\left(a^{2}\right)$ discretization errors. At a fixed temperature, since $T=1 /\left(a \cdot N_{\tau}\right)$, the continuum is reached by extrapolating in $1 / N_{\tau}^{2}$, so

$$
m_{N_{\tau}}=m^{\text {cont. }} \cdot\left(1+d \cdot \frac{1}{N_{\tau}^{2}}\right)
$$

Higher orders as $\mathrm{O}\left(a^{4}\right)$ or $\mathrm{O}\left(a^{2} \ln (a)\right)$ could enter into eq. (7.1), but these contributions seem to be small enough to not influence the results. 

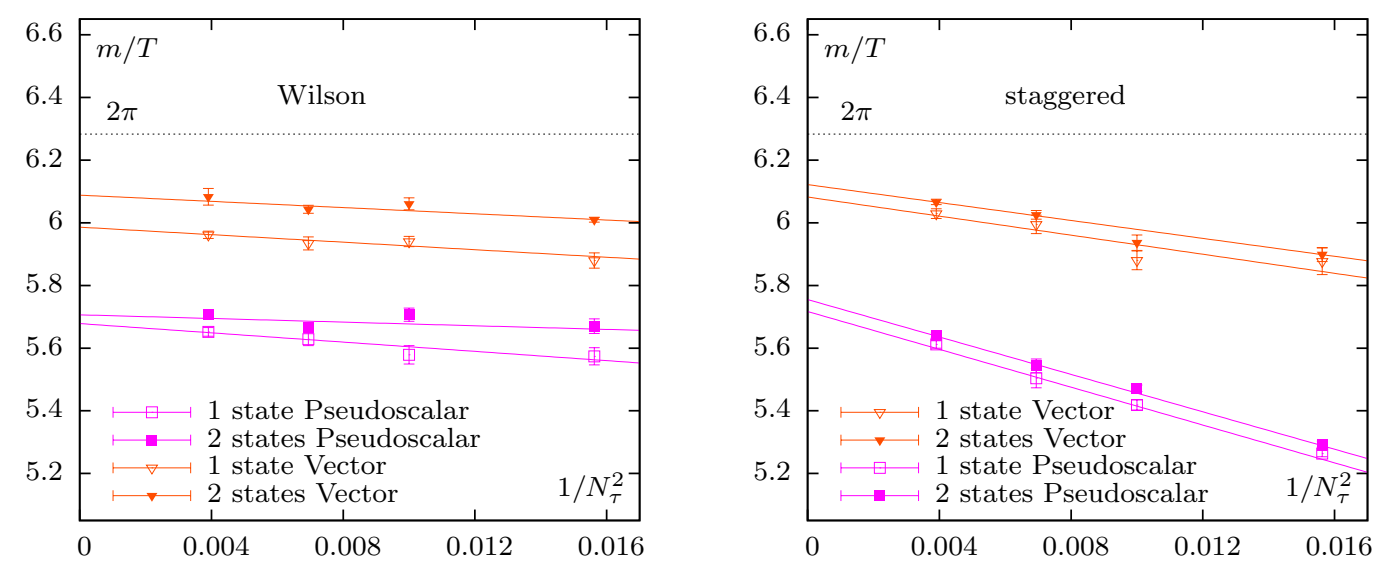

Figure 4: Continuum extrapolation using eq. (7.1) of the pseudoscalar and vector channels, for both Wilson (left) and staggered (right) fermions, at $1.5 T_{c}$. The data points are the result of the thermodynamic limit (infinite volume extrapolation of the one-state fits) and the ground state masses obtained from two-state fits. The dotted line marks the free theory mass of $2 \pi T$.

The extrapolation results are shown in fig. 4. Comparing Wilson and staggered fermions in these plots, it becomes very apparent that the staggered action is much more strongly dependent on the lattice spacing, while Wilson fermions seem much less affected.

\section{Results and conclusions}

The final results of this study are meson screening masses extrapolated to the thermodynamic and continuum limit, for the pseudo-scalar, scalar, vector and axial-vector channel, for both the Wilson and the staggered action, and at both temperatures $1.5 T_{c}$ and 3.0 $T_{c}$. In fig. 5 all final data points are summarized.

The differences between both actions on finite lattices vanish when the ground state masses are extracted (by taking the thermodynamic limit or performing a two-state fit) and the continuum limits are carried out. The staggered action shows much more pronounced lattice spacing effects, so the continuum limit is a crucial step. Finite lattice volume effects are similar for both actions.

The thermodynamic limit can be reached by extrapolation through ansatz eq. (5.1) or by fitting multiple states to a correlator on a reasonably sized lattice. Except for the Wilson (axial)vector, these two-state fits reach results compatible to the full thermodynamic limit, when carried out at aspect ratios of $N_{\sigma} / N_{\tau} \geq 4$ and at temporal extends $N_{\tau} \geq 12$. If this holds for other temperatures and actions, it might help to reduce the lattice sizes and thereby computing cost needed in screening masses studies.

Both the pseudoscalar and the scalar masses as well as the vector and the axialvector mass are clearly degenerate at both temperatures $1.5 T_{c}$ and 3.0 $T_{c}$. This holds not only for the continuum extrapolation, but already for finite size lattices.

HTL and DR results $[1,2]$ currently predict the free theory meson screening masses $2 \pi T$ to be reached from above, for both the (pseudo)scalar and the (axial)vector. At $1.5 T_{c}$, none of the 

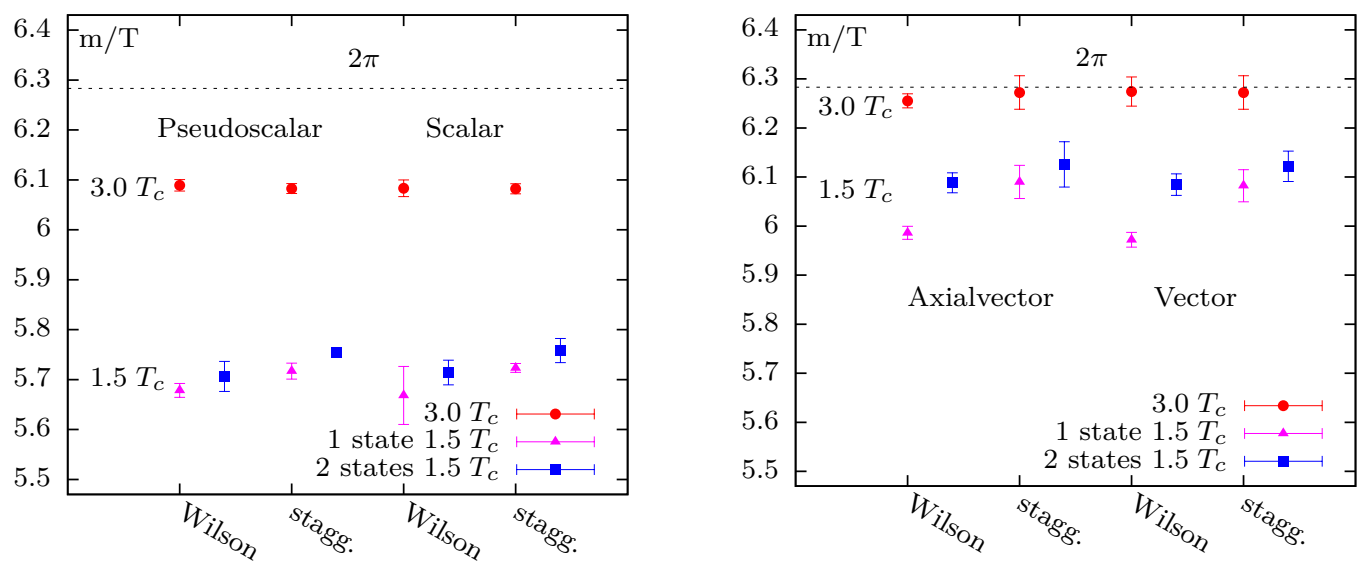

Figure 5: Continuum limit for the (pseudo)scalar and (axial)vector for both the Wilson and the staggered action. At $1.5 T_{c}$, both the masses from the thermodynamic limit (1 state fits) and the ground state mass from two-state fits have been extrapolated to the continuum. At 3.0 $T_{c}$, masses have only been extrapolated from the thermodynamic limit.

masses reach this free theory limit, they are both clearly below. For 3.0 $T_{c}$, all masses are closer to $2 \pi T$ and the vector and axial-vector are compatible with $2 \pi T$.

The free theory also predicts a full degeneracy between scalar, vector, pseudoscalar and axialvector, so a common mass for all four channels should be seen when approaching the free limit. This is not observed at either temperature.

\section{Acknowlegdements}

The results have been achieved by using JUGENE resources at the Jülich Supercomputing Centre and GPU-cluster resources of the lattice gauge theory group at Bielefeld University. This work is supported by the IRTG/GRK 881 "Quantum Fields and Strongly Interacting Matter".

\section{References}

[1] W. Alberico, A. Beraudo, A. Czerska, P. Czerski, and A. Molinari, Meson Screening Masses in the Interacting QCD Plasma, Nucl.Phys. A792 (2007) 152, [hep-ph / 0703298 ].

[2] M. Vepsalainen, Mesonic screening masses at high temperature and finite density, JHEP 0703 (2007) 022, [hep-ph/0701250].

[3] M. Cheng, S. Datta, A. Francis, J. van der Heide, C. Jung, et al., Meson screening masses from lattice QCD with two light and the strange quark, Eur.Phys.J. C71 (2011) 1564, [arXiv: 1010.1216$].$

[4] S. Gupta and N. Karthik, Hadronic Screening in Improved Taste, Phys.Rev. D87 (2013) 094001, [arXiv:1302.4917].

[5] M. Luscher, S. Sint, R. Sommer, P. Weisz, and U. Wolff, Nonperturbative O(a) improvement of lattice QCD, Nucl.Phys. B491 (1997) 323, [hep-lat/ 9609035$].$

[6] M. Fukugita, H. Mino, M. Okawa, G. Parisi, and A. Ukawa, Finite size effect for hadron masses in lattice QCD, Phys.Lett. B294 (1992) 380, [DOI: $10.1016 / 0370-2693$ (92) 91537-J]. 